\title{
Aortic Root Replacement for Pseudoaneurysm Arising from Freestyle Aortic Bioprosthesis
}

\author{
Mario Castillo-Sang, $\mathrm{MD}^{1}$, Rochus Voeller, $\mathrm{MD}^{2}$, Marc R. Moon, $\mathrm{MD}^{2 *}$ \\ ${ }^{1}$ Division of Cardiothoracic Surgery, Medical University of South Carolina, Charleston, South Carolina, USA; and ${ }^{2}$ Division of Cardiothoracic \\ Surgery, Washington University School of Medicine in St. Louis and Barnes-Jewish Hospital, St. Louis, Missouri, USA
}

\begin{abstract}
A 70-year-old female presented with a new systolic murmur and shortness of breath three years after undergoing aortic root replacement using a 27-mm Medtronic Freestyle $^{\circledR}$ stentless full root bioprosthesis (Medtronic, Inc., Fridley, Minnesota, USA). A large complex aortic root pseudoaneurysm was identified on contrasted computed tomography of the chest and transesophageal echocardiogram. We describe the redo aortic root replacement using a customized Dacron tube/valve composite graft with a proximal "skirt" and a modified Cabrol technique.

Copyright $\odot 2014$ Science International Corp.
\end{abstract}

\section{Key Words}

Aortic root - Aortic valve replacement $\cdot$ Freestyle $\cdot$ Root pseudoaneurysm - Xenograft $\cdot$ Cabrol technique

\section{Introduction}

Aortic root pseudoaneurysms are uncommon but highly morbid complications of the Medtronic Freestyle ${ }^{\circledast}$ aortic root bioprosthesis (Medtronic, Inc., Minneapolis, MN, USA) and their repair typically entails a technically difficult aortic root replacement, given adhesions and loss of tissue planes around the coronary buttons and coronary arteries. In confronting root pseudoaneurysms one has to rely on preoperative imaging and have a good primary and secondary plan for managing the aortic root. We present our approach to managing an aortic root pseudoaneurysm originating from a porcine xenograft.

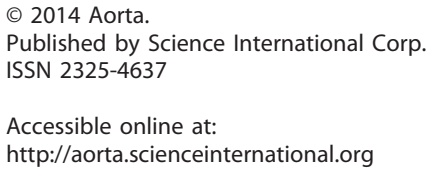

\section{Case Presentation}

A 70-year-old Caucasian female presented with new-onset systolic murmur and shortness of breath three years after undergoing aortic root replacement using a 27-mm Medtronic Freestyle ${ }^{\circledR}$ stentless bioprosthesis (Medtronic, Fridley, Minnesota) for annular dilation and severe aortic regurgitation.

Computed tomography (CT) with intravenous contrast revealed a large complex pseudoaneurysm of the aortic root; transesophageal echocardiogram confirmed the findings and demonstrated severe aortic regurgitation (Fig 1). The pseudoaneurysm had two components: one originating anteriorly near the right sinus of Valsalva measuring $3 \times 1.5 \times 2.5 \mathrm{~cm}$, and the second originating more posteriorly in the noncoronary sinus of Valsalva and into the left sinus near the origin of the left main coronary artery measuring $2.4 \times 0.8 \times 1.8 \mathrm{~cm}$ (Fig. 2). The overall diameter of the aortic root including the pseudoaneurysm was $6.5 \mathrm{~cm}$. The patient underwent reoperative aortic root replacement with complete excision of the Freestyle ${ }^{\circledR}$ stentless bioprosthesis and implantation of a $28-\mathrm{mm}$ Dacron Hemashield tube graft (Meadox Medicals, Inc., Oakland, New Jersey, USA).

\section{Surgical Technique}

Cannulation for cardiopulmonary bypass was performed via a right axillary artery anastomosis to an 8-mm Dacron Hemashield tube graft (Meadox Medi-

*Corresponding author:

Marc R. Moon, MD

Division of Cardiothoracic Surgery

Washington University School of Medicine in St. Louis

St. Louis, Missouri 63110, USA

Tel: +1 314362 6237, Fax: +1 314747 0917. E-Mail: moonm@wudosis.wustl.edu 


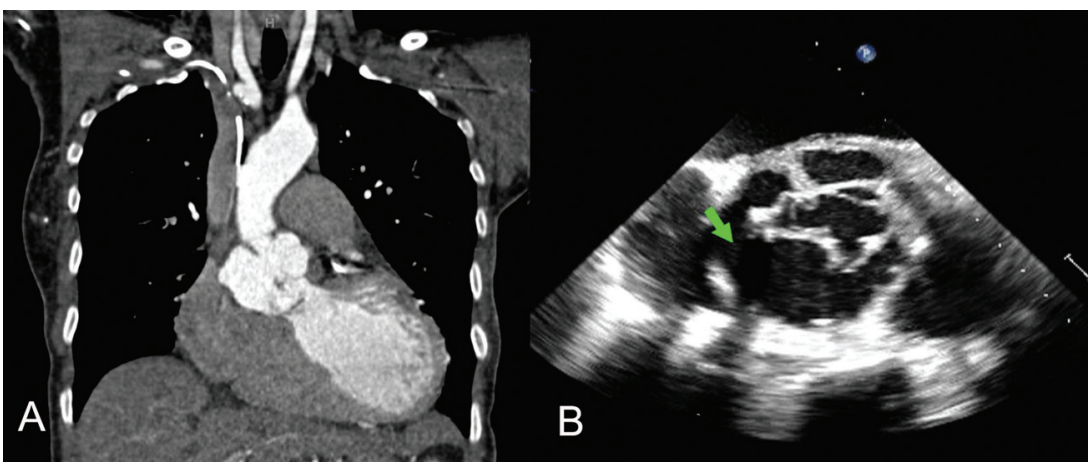

Figure 1. A. Computed tomography of chest (coronal view) with intravenous contrast showing a pseudoaneurysm of the aortic root. B. Transesophageal ultrasound showing the pseudoaneurysm of the aortic root and the perforations marked by the arrow.

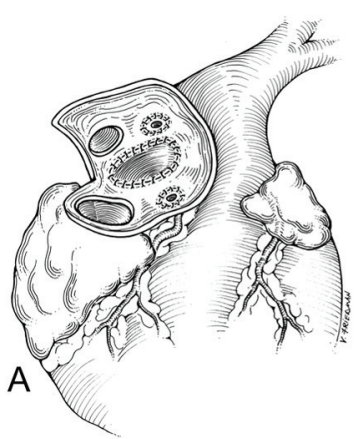

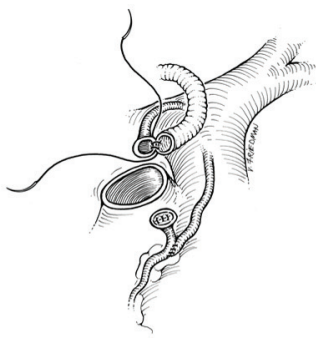

B
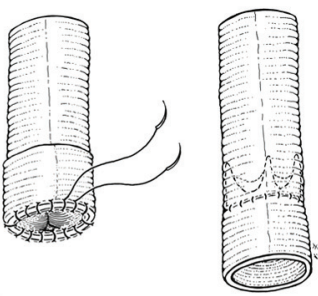

C
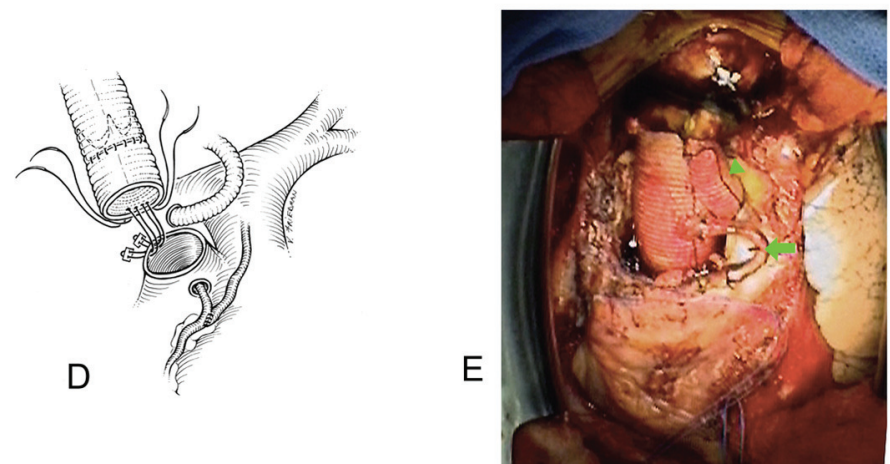

Figure 2. A. Artist's rendering showing the two perforations of the porcine homograft. B. Artist's rendering showing the coronary buttons, and the 12-mm Dacron graft anastomosis to the left coronary button and saphenous vein graft to the right coronary artery. C. Artist's drawing showing the creation of a stented valve-Dacron conduit by sewing a bioprosthetic valve into the Dacron graft that has been folded over itself. Once the folded Dacron graft is straightened it creates a skirt to anastomose to the aortic root. D. Anastomosis of the composite Dacron/valve graft to the aortic root using mattress-pledgeted sutures. E. Intraoperative picture showing the final appearance of the replaced aortic root. The arrowhead marks the left main coronary artery Cabrol anastomosis to the neoaorta. The arrow marks the saphenous vein graft to the right coronary artery proximal anastomosis to the neoaorta.

cals, Inc, Oakland, New Jersey, USA) to which the arterial cannula was connected and a long femoral venous cannula for drainage positioned using transesophageal echo guidance, with the tip at the junc- tion of the superior vena cava and right atrium. The chest was reentered using an oscillating saw and a segment of right saphenous vein was harvested for possible coronary reconstruction. 
Following extensive adhesiolysis, the aorta was cross-clamped immediately proximal to the innominate artery. The heart was arrested with antegrade cardioplegia and the arrest was maintained with intermittent retrograde cardioplegia, accompanied by topical cooling. The aorta was transected in the mid ascending portion, allowing us to confirm that the distal aorta was intact. Inspection of the root showed that the valve had been destroyed by presumed previous endocarditis, although there were no signs of active infection and intraoperative Gram's stain and cultures were negative. Inspection of the Freestyle ${ }^{\circledR}$ stentless bioprosthesis confirmed our preoperative CT scan findings of a large wall defect in the noncoronary sinus and a second defect in the right coronary sinus. The right and noncoronary leaflets of the bioprosthesis were almost completely unhinged and prolapsing into the ventricle leading to aortic regurgitation. The Freestyle ${ }^{\circledR}$ stentless bioprosthesis was removed completely while preserving both coronary buttons (Fig. 2). Dissection of the left main coronary artery could not be performed beyond the ostium because of its dense adhesions to the pulmonary artery, so a modification of the Cabrol technique with an end-to end anastomosis of the left button to a $12-\mathrm{mm}$ woven Dacron Hemashield tube graft was used (Fig. 2). The right coronary ostium was densely adhered to its surrounding tissues; thus, a saphenous vein graft was anastomosed end-to-side to the right coronary $2 \mathrm{~cm}$ distal to the ostium and the ostium was then oversewn with a 4-0 polypropylene suture (Fig. 2).

The end of a 28-mm Dacron Hemashield tube graft (approximately $1.5 \mathrm{~cm}$ ) was everted/folded over itself in an elephant trunk fashion to create a proximal "skirt." A 21-mm Magna valve was sewn to the edge of the everted portion of the graft, initially with three simple tacking sutures at 120-degree intervals, then with a running 4-0 polypropylene circumferentially (Fig. 2). The graft "skirt" was then rolled down providing $1 \mathrm{~cm}$ of Dacron graft proximal to the valve (Fig. 2). A total of 18 2-0 pledgeted, braided polyester sutures were brought outside to inside of the ventricular outflow tract with no gaps between. The annular sutures were then brought through the Dacron skirt below the bioprosthetic valve from inside to outside to attach the graft, and tied to secure the composite valve-graft to the ven-

Castillo-Sang, M. et al. tricular outflow tract (Fig. 2). The distal anastomosis was performed using an outside felt buttress and 3-0 polypropylene in a running fashion. The left main conduit was measured to reach the anterior aspect of the neoaorta from the left side, as this provided a smooth pathway for reconstruction and was anastomosed to the 28-mm Dacron graft using a 4-0 polypropylene suture (Fig. 2, arrowhead). The saphenous vein graft proximal anastomosis to the neoaorta was performed in a standard end-to-side fashion (Fig. 2, arrow). With the reconstruction completed, we placed a root vent in the neoaorta and de-aired the heart.

Total cross-clamp time was 2 hours and 17 minutes. The patient had an uneventful postoperative period and was discharged on postoperative day 10 . Empiric intravenous antibiotic therapy was continued for 6 weeks postoperatively because of presumed culture-negative endocarditis. The patient was found to be doing well at 1-month and 6-month follow-up.

\section{Discussion}

We describe the repair of an aortic root pseudoaneurysm arising from a Medtronic Freestyle ${ }^{\circledR}$ porcine graft (Medtronic, Fridley, Minnesota, USA) secondary to primary graft perforation in two areas. Our repair consisted of replacing the aortic root and reimplanting the left main and right coronary arteries. Previous reports have described pseudoaneurysm formation after anastomotic dehiscence [1]; others encountered true graft deterioration and perforation [2,3], and even traumatic disruptions have been described [4]. Isolated pseudoaneurysms of coronary buttons can be repaired using the technique previously described by Schmoker and Miller [5], but in the current case, the valve needed to be replaced as well. Noncoronary sinus of Valsalva perforations and pseudoaneurysms have been described $[2,3]$, with one report identifying two defects in the biograft itself [3], as we did. Repair of the perforation has been successfully done in the absence of infection [5] but usually requires an aortic root replacement. We elected to perform a Cabrol-type reimplantation of the coronary arteries given the dense adhesions impeding their dissection and hindering their length. A valve-Dacron 
graft conduit was created with a proximal skirt to facilitate proximal graft anastomosis and allow the aorto-ventricular junction to retain its flexible nature without myocardium contracting against a rigid structure, thus serving as a shock absorber and improving hemostasis. This skirted Dacron graft technique would also allow a future aortic valve replacement to be performed without the need to perform a root replacement by simply cutting the running and the three tacking sutures holding the bioprosthesis in place.

\section{Acknowledgment}

We acknowledge the assistance of Vicky Friedman at MedPIC Medical Illustration for the drawings.

\section{Conflict of Interest}

The authors have no conflict of interest relevant to this publication.

\section{Comment on this Article or Ask a Question}

\section{References}

1. Kucher N, Kipfer B, Seiler C, Allemann Y. Giant anastomotic pseudoaneurysm complicating aortic xenograft replacement. Ann Thorac Surg. 2004;77:2197-2199. 10.1016/S00034975(03)01269-4

2. Kitamura A, Handa N, Shomura Y, Wakiyama $H_{\text {, }}$ Shinka M, Fujiwara $\mathrm{H}$, et al. Reoperation for early failure of a freestyle bioprosthesis using a full root technique. J Heart Valve Dis. 2007;16:275277.

3. Ozaki N, Hino $Y$, Hanafusa $Y$, Yamashita $T$, Okada K, Tsukube T, et al. Perforation of the
Valsalva sinus after implantation of Medtronic Freestyle aortic bioprosthesis. Ann Thorac Surg. 2006;82:2282-2285. 10. 1016/j.athoracsur.2006.04.074

4. Albacker T, Al Kindi A, de Varennes B. Traumatic dehiscence of Medtronic Freestyle stentless bioprosthesis. Ann Thorac Surg. 2008;86:643-645. 10.1016/j.athoracsur.2008. 01.092

5. Schmoker JD, Miller D. A simple trick for repairing coronary pseudoaneurysm complicating a Bentall operation. Ann Thorac
Surg. $2002 ; 74: 268-270$. 10.1016/S00034975(02)03543-9

Cite this article as: Castillo-Sang $\mathrm{M}$ Voeller R, Moon MR. Aortic Root Replacement for Pseudoaneurysm Arising from Freestyle Aortic Bioprosthesis. Aorta 2014;2(5):202-206. DOI: http://dx.doi. org/10.12945/j.aorta.2014.14-034

\section{EDITOR'S COMMENTS AND QUESTIONS}

\section{Editor's Comments}

This superb case report from Moon and colleagues illustrates several important points about reoperative surgery on the aortic root, including the following:

- The importance of arterial and venous access outside the chest in cases of root pseudoaneurysm.

- The fact that the coronary buttons are often nonmobilizable in reoperative cases.

- The usefulness of the modified Cabrol (Dacron graft) technique for coronary button reattachment in such cases.

- The handy elephant trunk/skirt technique to facilitate the proximal graft anastomosis.

\section{Editor's Questions}

1. Why do you think your case and those in your references developed these defects in the wall of the Freestyle graft? Do you have any idea what is the underlying pathology?
We highly suspect that in our case the structural deterioration to the graft was caused by infection although we could not prove this with blood cultures or cultures of the graft material. Our patient was, nonetheless, managed with an 8-week course of intravenous antibiotics. Other case reports suggest there might be a weakness to the Freestyle graft at the noncoronary sinus of Valsalva. Pseudoaneurysms of the coronary buttons have also been reported.

2. Are you concerned that your 12-mm graft may be too large for a single coronary button? Are you concerned that the peripheral, nonflowing portion of the lumen of the $12-\mathrm{mm}$ graft may develop thrombus in the future?

The left main coronary was large and we wanted to match the button size with a graft that would provide high flows and so the 12-mm graft was chosen. One can argue that a $10-\mathrm{mm}$ graft would have had a similar result. The peripheral, nonflowing portion of the graft was imbricated on itself and sutured in order to eliminate the nonflowing lumen. This is not very clear from our intraoperative picture, but on closer look at the central dark line of the Dacron graft one can make the curvature as the 
blind end is folded downwards and to the right of the patient.

3. Do you always route the Cabrol graft to the left of the main graft? Some surgeons bring it around to the right.
We would not. In this particular case the location and orientation of the ostia of the left main button pointed to the left of the patient and this particular layout afforded a smooth pathway without kinks in the graft or the anastomoses. 